\title{
Antimicrobial prophylaxis for transrectal ultrasound-guided prostate biopsy: fosfomycin trometamol, an attractive alternative
}

Article in World Journal of Urology · May 2016

DOI: $10.1007 / 500345-016-1867-6$

\section{CITATIONS}

3

21 authors, including:

\section{Mauro Gacci}

University of Florence

255 PUBLICATIONS $\quad$ 4,029 CITATIONS

SEE PROFILE

\section{Carlo Trombetta}

Università degli Studi di Trieste 283 PUBLICATIONS 1,011 CITATIONS SEE PROFILE
READS

74

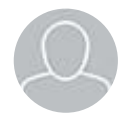

\section{Giovanni Liguori}

Università degli Studi di Trieste

169 PUBLICATIONS 929 CITATIONS

SEE PROFILE

\section{Riccardo Bartoletti}

Università di Pisa

165 PUBLICATIONS 1,612 CITATIONS

SEE PROFILE

Some of the authors of this publication are also working on these related projects:

Optimized instillation therapy in NMIBC for intermediate and high risk Patients with radiofrequency induced thermochemotherapeutic effect "RITE" based on the Synergo system View project 


\title{
Antimicrobial prophylaxis for transrectal ultrasound-guided prostate biopsy: fosfomycin trometamol, an attractive alternative
}

\author{
Tommaso Cai ${ }^{1} \cdot$ Luca Gallelli $^{2} \cdot$ Andrea Cocci $^{3} \cdot$ Daniele Tiscione $^{1} \cdot$ \\ Paolo Verze $^{4} \cdot$ Michele Lanciotti $^{3} \cdot$ Davide Vanacore $^{3} \cdot$ Michele Rizzo $^{5}$.

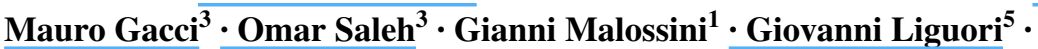 \\ Carlo Trombetta $^{5} \cdot$ Damiano Rocco $^{6} \cdot$ Alessandro Palmieri $^{4} \cdot$ Riccardo Bartoletti $^{7}$. \\ Marco Carini $^{3} \cdot$ Florian M. E. Wagenlehner ${ }^{8} \cdot$ Kurt Naber $^{9} \cdot$ Vincenzo Mirone $^{4}$. \\ Truls E. Bjerklund Johansen ${ }^{10}$
}

Received: 3 February 2016 / Accepted: 23 May 2016

(C) Springer-Verlag Berlin Heidelberg 2016

\begin{abstract}
Objective To compare fosfomycin trometamol (FT) and ciprofloxacin (CIP) for antibiotic prophylaxis in transrectal prostate biopsy (TR-PB).

Patients and methods Data for 1109 patients (mean age $66.7 \pm 8.45$ ) who underwent TR-PB between March to September 2015 in seven Italian urological institutions were retrospectively reviewed, of which 632 received FT (Group 1) and 477 received CIP (Group 2) for prophylaxis. We reviewed all urine culture results obtained after the procedure, all adverse drug reactions (ADRs) related to the drug and all febrile and/or symptomatic urinary tract
\end{abstract}

Tommaso Cai

ktommy@libero.it

1 Department of Urology, Santa Chiara Regional Hospital, Largo Medaglie d'Oro, 9, 38123 Trento, Italy

2 Department of Health Science, School of Medicine, University of Catanzaro, Catanzaro, Italy

3 Department of Urology, University of Florence, Florence, Italy

4 Department of Urology, University of Naples, Federico II, Naples, Italy

5 Department of Urology, University of Trieste, Trieste, Italy

6 Department of Urology, University of Catanzaro, Catanzaro, Italy

7 Department of Urology, University of Pisa, Pisa, Italy

$8 \quad$ Klinik und Poliklinik für Urologie, Kinderurologie und Andrologie, Universitätsklinikum Giessen und Marburg GmbH, Justus-Liebig-Universität Giessen, Giessen, Germany

9 Department of Urology, Technical University of Munich, Munich, Germany

10 Department of Urology, Oslo University Hospital, Oslo, Norway infections (UTIs) occurring within 1 month after TR-PB. The rate of symptomatic UTIs and the rate of ADRs were considered the main outcome measures.

Results In the total study population, 72/1109 (6.5\%) patients experienced symptomatic UTIs and among these $11(0.9 \%$ of total) had urosepsis. Out of 72, $53(73.6 \%)$ symptomatic UTIs were caused by fluoroquinolone-resistant strains. Out of 632, $10(1.6 \%)$ patients in Group 1 and 62/477 (12.9\%) patients in Group 2 had symptomatic UTIs $(p<0.001)$; in particular, $2 / 632(0.3 \%)$ patients in Group 1 and 9/477 (1.8\%) patients in Group 2 had urosepsis $(p<0.001)$. No differences were reported in terms of adverse events ( 0.6 vs $0.4 \% ; p=0.70$ ). A Charlson comorbidity index $\leq 1$ and type of antimicrobial prophylaxis (FT) were found to be associated with a lower probability of symptomatic UTIs in the multivariate model.

Conclusions Antibiotic prophylaxis with FT for TR-PB had a lower rate of adverse events and a lower rate of symptomatic UTIs as compared with CIP. Fosfomycin trometamol appears as an attractive alternative prophylactic regimen in prostate biopsies.

Keywords Prostate biopsy · Fosfomycin trometamol · Fluoroquinolone $\cdot$ Prophylaxis $\cdot$ Transrectal ultrasonography $\cdot$ Urinary tract infection

\section{Introduction}

Prostate biopsy is currently an indispensable method for the diagnosis of prostate cancer, and the transrectal approach is most commonly used by European urologists [1-3]. Even if transrectal biopsy of the prostate (TR-PB) is generally considered a safe method, it may be accompanied by severe clinical complications, ranging from 
bleeding due to the biopsy trauma to infectious complications including asymptomatic bacteriuria, symptomatic urinary tract infections (UTIs) and sepsis [4]. In recent years, a higher rate of infectious complications after TR-PB has been reported [5]. The rate is correlated with increasing antimicrobial resistance, especially to fluoroquinolones which are the current first-line recommended antibiotics. In fact, a dramatic increase in ciprofloxacinresistant strains among Escherichia coli with decreasing efficacy of ciprofloxacin prophylaxis in patients undergoing TR-PB has been reported [6]. The current rate of fluoroquinolone-resistant organisms has become a significant health problem [3]. The situation is due both to over-prescription of antibiotics and the limited number of available, especially oral antibiotics for prophylaxis in TR-PB. Furthermore, there are no new antibiotics in the pipelines of the pharmaceutical companies to replace oral fluoroquinolones in the near future [3, 7]. Thus, infectious complications after TR-PB represent an important challenge for the urologist and for patients. On the other hand, several authors reported that fosfomycin trometamol (FT) could be a valid alternative for antibiotic prophylaxis of TR-PB due to its low profile of resistance, elevated activity against multidrug-resistant (MDR) Gram-negative bacteria and favorable pharmacokinetic properties [8-10]. Here, we aimed to compare the clinical outcome of patients who underwent TR-PB and received prophylaxis with either FT or ciprofloxacin (CIP), by means of a retrospective, comparative cohort study.

\section{Patients and methods}

\section{Study design and population}

Our study population consisted of a cohort of patients who underwent TR-PB in 7 tertiary referral urological institutions from January to September 2015. Each trialist was asked to select all consecutive patients who had received antibiotic prophylaxis for prostate biopsy with FT or CIP during the 9-month study period. Clinical and microbiological data were collected by reviewing all electronic medical charts and, if needed, by reviewing medical ward charts or by calling the patients. All collected patients were dived into two groups. Group 1: patients who received a dose of $3 \mathrm{~g}$ FT orally $3 \mathrm{~h}$ before and $3 \mathrm{~g} 24 \mathrm{~h}$ after the first administration (in line with the registration recommendations); Group 2: all patients who received $500 \mathrm{mg}$ CIP as prophylaxis administered orally twice daily for 5 days starting 1 day before the procedure.

In Group 2, we decided to collect data only from patients who had undergone CIP prophylaxis for the following reasons:
- CIP is the most common antibiotic prescribed in Italy for TR-PB prophylaxis.

- We wanted to obtain a group of patients as homogenous as possible for comparison and further analysis.

Rate of symptomatic UTIs was the primary end point. All clinical and microbiological data were compared between the two groups. Adverse drug reactions (ADRs) were the secondary end points and were registered according to the Clavien-Dindo classification [11].

\section{Ethical considerations}

The present study has a retrospective cohort design. The medical records of groups of individuals who are alike in many ways but differ by a certain characteristic (patients receiving antibiotic prophylaxis with FT versus CIP) are compared for a particular outcome (rate of symptomatic UTIs) [12]. The study was conducted in line with the STROBE statement (http://www.strobe-statement.org). Figure 1 shows the study flowchart. Due to the retrospective nature and due to the fact that FT has been registered in Italy for diagnostic urological procedures, the study did not require approval by the local ethics committee (IRB). All anamnestic, clinical and laboratory data containing protected patient's health information were de-identified in order to use anonymous data for analysis. The de-identification was performed by non-medical staff by means of dedicated software. Trialists did not process or analyze data until after the de-identification process.

\section{Inclusion and exclusion criteria}

We included all patients older than 18 years and candidates for prostate biopsy, in line with the indications of the European Association of Urology (EAU) [13]. Subjects affected by significant comorbidities (Charlson comorbidity index >3) [14] and with known anatomical abnormalities of the urinary tract were excluded from the study. In order to obtain as homogenous cohorts of patients as possible, the microbiological history of all patients was evaluated and all patients who reported previous symptomatic UTIs due to fluoroquinolone-resistant and fosfomycin-resistant strains were excluded.

\section{Biopsy procedure}

Before TR-PB, all patients in this retrospective analysis underwent the following procedure: dipstick urinalysis (leukocyte esterase test and nitrite test) and/or microscopic urinalysis and urine culture. A urine culture taken before the procedure was mandatory for inclusion. All patients received a self-administered fleet enema (sodium 
Fig. 1 The study flow chart

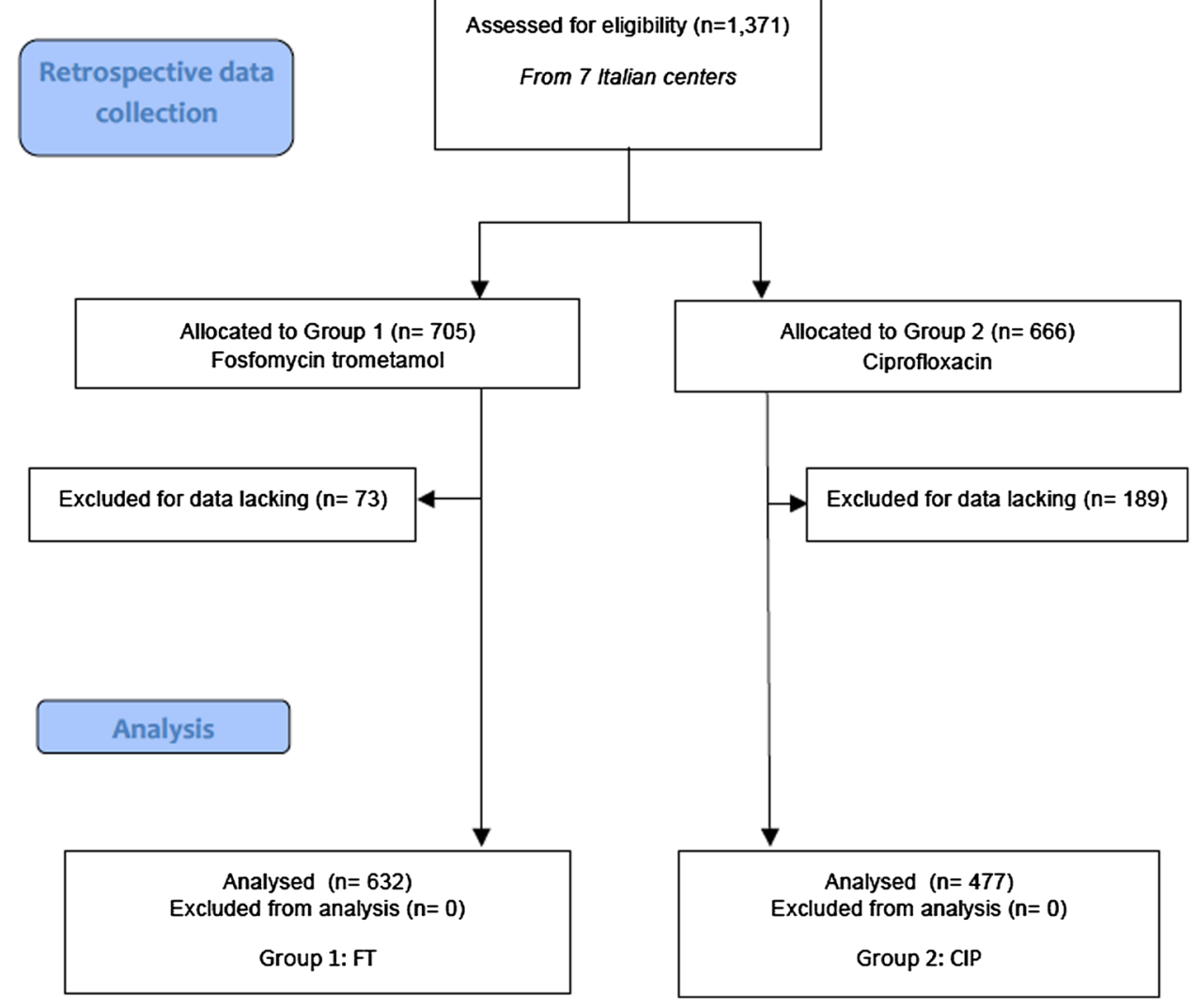

phosphate and dibasic sodium phosphate) $2 \mathrm{~h}$ before the biopsy. At the procedure time, the microbiological results were reviewed and all patients with a positive urine culture were excluded if not treated before. In patients who had been treated before, a negative microbiological control after therapy was required before biopsy. With the patient in the left-lying position, the urologist performed transrectal ultrasound with a multiplanar multifrequency probe attached to the ultrasound scanner. Before prostate biopsy, local anesthesia was administered transrectally. Prostate biopsies (12 cores) were taken using an automated biopsy gun with a disposable 16 gauge x $25 \mathrm{~cm}$ biopsy needle. All intra-procedural complications were recorded, and all patients were instructed to return to the emergency department of the same hospital if they developed complications such as fever, chills, macroscopic hematuria or severe voiding symptoms. A follow-up visit for discussing histological results of the prostate biopsy and for evaluation of possible complications was scheduled 30 days after the procedure. Only patients who also had a urine culture at the follow-up visit were included.

\section{Data collection and microbiological considerations}

The following data were recorded retrospectively: age, Charlson comorbidity index, microbiological results of a midstream-voided urine before TR-PB, date of TR-PB, patient-related and external risk factors for UTI development, PSA level, results of the digital rectal examination, IPSS and IIEF-5 questionnaires before the procedure (as it is routine in each urological institution), the presence of adverse events related to the antibiotics or to the procedure, Clavien-Dindo complication score, microbiological results of a midstream-voided urine after TR-PB and histological findings. We registered all episodes of symptomatic UTIs that occurred within 1 month after the procedure. A 1-month interval was chosen to increase the likelihood of capturing all symptomatic UTIs attributable to the prostate biopsy [15]. Data were collected by reviewing all ambulatory and hospital medical charts in case of hospitalization for symptomatic UTIs. In line with EAU guidelines, we defined an episode of symptomatic UTI as follows [16-18]:

- body temperature $<38{ }^{\circ} \mathrm{C}$ and dysuria accompanied by pyuria.

- Fever $\geq 38{ }^{\circ} \mathrm{C}$ accompanied by one symptom of the lower urinary tract (i.e., urgency, frequency, dysuria, or suprapubic tenderness).

- Fever $\geq 38{ }^{\circ} \mathrm{C}$, accompanied by chills and malaise

- urosepsis as defined in the EAU guidelines on urological infections [18]. 
The classification of peri-procedural complications was performed using the Clavien-Dindo classification [11]. Although symptomatic UTIs confirmed by microbiological analysis were the main outcome parameter in this study, all other symptoms were also recorded and considered. All bacterial isolates were identified by standard microbiological methods according to Hooton et al. [19], and susceptibility testing was performed according to CLSI (Clinical and Laboratory Standards Institute) recommendations. All MIC values of isolates were interpreted according to the breakpoints of European Committee on Antimicrobial Susceptibility Testing (EUCAST) [20]. All institutions followed these criteria. For microbiological diagnosis of UTI, a cutoff of $\geq 10^{5}$ colony-forming units $/ \mathrm{mL}$ were used in all institutions.

\section{Questionnaires}

The validated Italian versions of the International Prostate Symptom Score (IPSS) [21] and International Index of Erectile Function (IIEF) [22] were administered to each patient, as it is routine in each urological institution. The questionnaire was offered to the patient on arrival at each center before the procedure and at the first follow-up visit after the procedure.

\section{Safety}

The safety of the antibiotics was assessed in terms of frequency and nature of ADRs. The association between ADRs and drug treatment was performed in agreement with Gallelli et al. [23] by means of the Naranjo Adverse Probability Scale [24]. Records positive for ADRs were reviewed by blinded clinicians, who simply looked for reported ADRs in clinical records and completed the Naranjo probability scale.

\section{Statistical analysis}

The Fisher's exact test or a two-tailed $t$ test or Mann-Whitney test was used to assess the statistical significance as appropriate. For the purpose of the case-control comparison between patients included in the two groups, all cases of symptomatic UTIs were matched on the basis of this criterion: similar age (age difference not exceeding \pm 5 years). All results have been adjusted by all risk factors for biopsy-related UTI complications, such as number of previous biopsy series. Continuous variables were given as the mean \pm standard deviation. Relative risks and $95 \%$ confidence intervals were estimated by applying log-binomial regression and Cox regression with a constant in the time variable. The following co-variables have been used in the multivariate analysis: previous UTIs (yes/no), Charlson comorbidity index (0/1/2), smoking (yes/no), repeat biopsy (yes/no), DRE-positive finding (yes/no) and type of antimicrobial prophylaxis (FT/CIP). Statistical significance was set at $p<0.05$. All reported $\mathrm{p}$ values are two sided. All statistical analyses were performed by using SPSS 11.0 for Apple Macintosh (SPSS Inc., Chicago, Illinois).

\section{Results}

In total, 1109 patients who had undergone TR-PB were included in this retrospective study, of which 632 received FT (Group 1) and 477 CIP (Group 2) for prophylaxis, respectively. No clinically significant infectious complications were found among patients excluded from the analysis [(Group 1: 73, Group 2: 189 (Fig. 1)] due to data lacking. All patients' clinical and laboratory characteristics at the time of the procedure are summarized in Table 1 . The two groups did not differ significantly in terms of clinical or other procedure-related parameters.

\section{Non-infectious prostate biopsy complications}

The most common recorded non-infectious complication was macrohematuria that was found in 442 patients in Group $1(69.9 \%)$ and in 338 in Group 2 (70.8\%), without any statistically significant difference $(p=0.78)$. All reported noninfectious complications are displayed in Table 2.

\section{Symptomatic UTIs after biopsy}

Seventy-two patients showed symptomatic UTIs (6.5\%): 10 out of 632 patients in Group 1 (1.6\%) and 62 out of 477 in Group $2(12.9 \%)(p<0.001)$.

\section{Group 1 (fosfomycin trometamol)}

In Group 1, we found 5 cases with afebrile UTIs $(0.8 \%)$ and 3 cases with febrile UTIs $(0.5 \%)$ not requiring hospitalization. These patients were treated with antibiotics in line with susceptibility testing. Furthermore, we found 2 cases of urosepsis $(0.3 \%)$ that required hospitalization and parenteral antibiotic therapy.

\section{Group 2 (ciprofloxacin)}

In Group 2, we found 25 cases with afebrile UTIs (5.2\%) and 28 cases with febrile UTIs (5.9\%) not requiring hospitalization. These patients were treated in line with susceptibility testing. Furthermore, 9 cases of urosepsis were registered $(1.8 \%)$.

The two groups were statistically significantly different in terms of symptomatic UTIs (1.6 vs $12.9 \%$; 
Table 1 Patient clinical and laboratory characteristics at the time of the prostate biopsy
Table 2 Clinical, histological data and non-infectious complication after prostate biopsy

\begin{tabular}{|c|c|c|c|}
\hline & & & $p\left(t\right.$ or $\left.\chi^{2} / d f\right)$ \\
\hline Group & 1 (Fosfomycin trometamol) & 2 (Ciprofloxacin) & \\
\hline No. of patients & 632 & 477 & \\
\hline Median age $( \pm \mathrm{SD})$ & $65.9( \pm 8.3)$ & $66.9( \pm 8.9)$ & $0.05(1.92 / 1107)$ \\
\hline Previous UTIs ${ }^{\mathrm{a}}$ & & & $0.59(0.28 / 1)$ \\
\hline Yes & $32(5.0)$ & $20(4.1)$ & \\
\hline No & $600(95.0)$ & 457 (95.9) & \\
\hline Charlson comorbidity index & & & $0.14(2.14 / 1)$ \\
\hline 0 & $598(94.6)$ & $461(96.6)$ & \\
\hline 1 & $32(5.0)$ & $16(3.4)$ & \\
\hline 2 & $2(0.4)$ & - & \\
\hline Rate of diabetes mellitus & $62(9.8)$ & $40(8.3)$ & $0.47(0.50 / 1)$ \\
\hline Smoking & & & $0.74(0.11 / 1)$ \\
\hline Yes & $284(44.9)$ & $220(46.1)$ & \\
\hline Body mass index $\left(\mathrm{Kg} / \mathrm{m}^{2}\right)( \pm \mathrm{SD})$ & $25.2( \pm 2.61)$ & $24.8( \pm 2.91)$ & $0.07(1.80 / 1107)$ \\
\hline PSA total $( \pm \mathrm{SD})$ & $7.14( \pm 4.31)$ & $7.69( \pm 5.09)$ & $0.05(1.94 / 1107)$ \\
\hline DRE-positive finding & $76(12.02)$ & $58(12.1)$ & $0.94(0.005 / 1)$ \\
\hline Repeat biopsy & $90(14.2)$ & $63(13.2)$ & $0.68(0.16 / 1)$ \\
\hline IPSS $( \pm \mathrm{SD})$ & $7.9( \pm 6.1)$ & $7.3( \pm 5.9)$ & $0.10(1.64 / 1107)$ \\
\hline IIEF-15 ( \pm SD $)$ & $17.9( \pm 3.5)$ & $18.1( \pm 2.9)$ & $0.31(1.01 / 1107)$ \\
\hline
\end{tabular}

Data in parentheses are percentages unless otherwise specified

$S D$ Standard deviation, UTIs urinary tract infection

${ }^{a}$ UTIs: yes/no due to fluoroquinolone-resistant strains

\begin{tabular}{|c|c|c|c|}
\hline & & & $p\left(t\right.$ or $\left.\chi^{2} / d f\right)$ \\
\hline Group & 1 (Fosfomycin trometamol) & 2 (Ciprofloxacin) & \\
\hline Histological findings & & & $0.76(0.08 / 1)$ \\
\hline Prostate cancer & $285(45.1)$ & $210(44.0)$ & \\
\hline $\mathrm{BPH}^{\mathrm{a}}$ & $332(52.5)$ & $251(52.6)$ & \\
\hline Normal prostate tissue & $15(2.4)$ & $16(3.4)$ & \\
\hline Adverse events related to the antibiotics & $4(0.6)$ & $2(0.4)$ & $0.94(0.004 / 1)$ \\
\hline \multicolumn{4}{|l|}{ Clavien-Dindo score } \\
\hline Grade 1 & $598(94.6)$ & $438(91.8)$ & $0.08(3.01 / 1)$ \\
\hline Macrohematuria & $442(73.9)$ & $338(77.1)$ & \\
\hline Hematospermia & $34(5.7)$ & $22(5.1)$ & \\
\hline Macrohematuria and Hematospermia & $60(10.1)$ & $41(9.4)$ & \\
\hline Dysuria & $62(10.3)$ & $37(8.4)$ & \\
\hline Grade 2 & $58(9.1)$ & $41(8.5)$ & $0.81(0.05 / 1)$ \\
\hline Acute urinary retention ${ }^{\mathrm{b}}$ & $41(70.6)$ & $36(87.8)$ & \\
\hline Rectal bleeding ${ }^{\mathrm{c}}$ & $17(29.4)$ & $5(12.2)$ & \\
\hline
\end{tabular}

Data in parentheses are percentages unless otherwise specified

a BPH, Benign Prostatic Hyperplasia

b Acute urinary retention: this complication required indwelling catheter for 1 week

${ }^{c}$ Rectal bleeding: this complication required a transrectal absorbable hemostatic gelatin sponge $p<0.001)$ and urosepsis $(0.3$ vs $1.8 \% ; p=0.02)$. The most common isolated strains from patients with symptomatic UTIs were as follows: E. coli, Enterococcus faecalis and Klebsiella spp. Fifty-three (73.6\%) symptomatic
UTIs were caused by fluoroquinolone-resistant strains. All clinical symptoms and microbiological findings in patients with symptomatic UTIs are displayed in detail in Table 3. 
Table 3 Symptomatic UTI complications and microbiological findings after prostate biopsy

\begin{tabular}{llll}
\hline & & & $p\left(\right.$ t or $\left.\chi^{2} / d f\right)$ \\
\hline Patients with symptomatic UTIs & $72(6.4)$ & & \\
Group & 1 (Fosfomycin trometamol) & 2 (Ciprofloxacin) & \\
Symptomatic UTIs & $10(1.6)$ & $62(12.9)$ & $<0.001(56.48 / 1)$ \\
Afebrile UTIs & 5 & 25 & \\
Febrile UTIs & 5 & 37 & $0.68(0.16 / 1)$ \\
Positive urine culture & $10(100)$ & $58(93.5)$ & $0.13(2.28 / 1)$ \\
Positive blood culture & $3(30)$ & $24(38.7)$ & $0.02(5.32 / 1)$ \\
Urosepsis (among all febrile UTIs) & $2(0.3)$ & $9(1.8)$ & $0.66(0.19 / 1)$ \\
Isolated bacteria strains & & & \\
Escherichia coli & $8(80)$ & $39(67.2)$ & \\
$\quad$ Fluoroquinolone-resistant E. coli & 6 & 15 & \\
$\quad$ ESBL E. coli & 3 & 9 & \\
Enterococcus faecalis & $1(10)$ & $8(13.9)$ & \\
Klebsiella spp. & $1(10)$ & & \\
\hline
\end{tabular}

Data in parentheses are percentages unless otherwise specified

Table 4 Univariate and multivariate analysis results of factors affecting of symptomatic UTIs development risk in all enrolled patients

\begin{tabular}{lll}
\hline Categories (variables) & Univariate analysis $(p)(\mathrm{HR} ; 95 \% \mathrm{CI})$ & Multivariate analysis $(p)(\mathrm{HR} ; 95 \%$ CI) \\
\hline Previous UTIs (yes/no) & $(0.66)(\mathrm{HR} 0.61 ; 0.14-1.25)$ & $(0.38)(\mathrm{HR} 0.82 ; 0.65-1.01)$ \\
Charlson comorbidity index $(0 / 1 / 2)$ & $(0.03)(\mathrm{HR} 2.92 ; 0.97-3.98)$ & $(<0.01)(\mathrm{HR} 3.49 ; 1.67-3.11)$ \\
Smoking (yes/no) & $(0.82)(\mathrm{HR} 0.52 ; 0.10-1.46)$ & $(0.66)(\mathrm{HR} 0.21 ; 0.08-1.17)$ \\
Repeat biopsy (yes/no) & $(0.09)(\mathrm{HR} 1.01 ; 0.22-1.54)$ & $(0.10)(\mathrm{HR} 1.25 ; 0.75-1.89)$ \\
DRE-positive finding (yes/no) & $(0.70)(\mathrm{HR} 0.32 ; 0.45-1.67)$ & $(0.69)(\mathrm{HR} 051 ; 0.20-1.14)$ \\
Type of antimicrobial prophylaxis (FT/CIP) & $(0.04)(\mathrm{HR} 2.43 ; 1.42-3.21)$ & $(<0.01)(\mathrm{HR} 2.67 ; 1.34-3.96)$ \\
\hline
\end{tabular}

$C I$ Confident interval, DRE digital rectal examination

\section{Multivariate analysis}

The Charlson comorbidity index $(\leq 1)$ and type of antimicrobial prophylaxis (FT) were found to be associated with a lower probability of symptomatic UTIs development in the multivariate model [ (Charlson comorbidity index $(\leq 1)$ $p=0.01 \mathrm{HR} 3.49$; $95 \% 1.67-3.11$; type of antimicrobial prophylaxis (FT) $p=0.01$ HR 2.67; $95 \%$ 1.34-3.96) (Table 4).

\section{Adverse drug reactions}

In Group 1, we found 4 mild adverse events $(0.6 \%)$ that did not require any additional treatment: 3 cases with diarrhea and 1 drug-related hives (treated with medical therapy). Also, in Group 2 we found a low prevalence of adverse events: 2 cases with diarrhea $(0.4 \%)$. No statistically significant difference between the two groups was observed in terms of adverse events (0.6 vs $0.4 \%$; $p=0.94)$.

\section{Discussion}

\section{Main findings}

Fluoroquinolones are recommended as the first choice for antibiotic prophylaxis in TR-PB by EAU [18] and AUA [25] guidelines, but in the last few years, the increasing resistance to fluoroquinolones has caused an alarming increase in severe infectious complications after prostate biopsy [26]. Recently, Wagenlehner et al. [3] reported that fluoroquinolones were used in $98.2 \%$ of patients, but resistance against fluoroquinolones was seen in $60 \%$ of all bacterial strains isolated after the procedure. An alternative strategy to the fluoroquinolone-based prophylaxis is therefore needed $[9,27]$. Here, we performed a retrospective cohort analysis in patients who underwent TR-PB and demonstrated that FT may be an attractive alternative antimicrobial prophylaxis for TR-PB due to a lower rate of symptomatic UTIs and a lower rate of adverse events as compared with CIP. 


\section{Comparisons with other studies}

Ongün et al. [15] evaluated the efficacy of single-dose FT prophylaxis in comparison with fluoroquinolone-based prophylaxis in patients undergone TR-PB. They found that among all patients with febrile UTI, 5.2 \% received prophylaxis with FT while $94.8 \%$ received fluoroquinolonebased prophylaxis [15]. In other words, FT seems to be an effective drug and a valid alternative for antimicrobial prophylaxis in TR-PB [15]. In 1998, Periti et al. [28] found, in a large cohort of patients undergoing transurethral resection of the prostate (TURP), that the incidence of significant postoperative bacteriuria in patients who received FT as prophylaxis was significantly lower than in the amoxicillin and cotrimoxazole groups. Recently, Rhodes et al. [27] determined the optimal administration time for peri-procedural prophylaxis with FT in an in vivo model. The authors evaluated the concentrations of FT in plasma, peripheral zone and transition zone of the prostate in 26 subjects undergoing TURP, following a single oral dose of $3 \mathrm{~g}$ of FT [27]. They recommended that for prophylaxis, FT should be given 1-4 h prior to prostate biopsy [27]. Moreover, they demonstrated that the concentrations of FT in the transitional zone of the prostate exceeded $4 \mathrm{mg} / \mathrm{L}$ for $90 \%$ of the population between 1 and $9 \mathrm{~h}$ after administration [27]. The concentrations of FT in the peripheral zone of the prostate were much lower and exceeded only $4 \mathrm{mg} / \mathrm{L}$ in $70 \%$ of the population between 1 and $4 \mathrm{~h}$ after administration [27]. In our study, we administered $3 \mathrm{~g}$ of FT $3 \mathrm{~h}$ before the procedure, in line with the findings in the Rhodes's study [27]; moreover, in accordance with the national registration recommendations we also gave a second dose after the procedure. Recently, Lista et al. [29] demonstrated, in a randomized prospective study on 671 patients who had undergone TR-PB, that antibiotic prophylaxis with FT (2 doses of $3 \mathrm{~g}$ ) is a good alternative in terms of efficacy and safety when compared with a long-term therapy with CIP (10 doses of $500 \mathrm{mg}$ ). Moreover, the use of FT is correlated with lower rate of resistance [29]. In the Lista's study, the urine culture was carried out at 2 weeks after biopsy [29], while in our study we collected a urine culture at 30 days from the procedure. We consider it advantageous to test the urine culture at 30 days for obtaining a longer microbiological follow-up period.

\section{Strengths and limitations of the present study}

Since the rate of severe febrile UTIs after prostate biopsy is still rather low, it is important to enroll a sufficiently high number of patients to demonstrate statistically significant differences as shown in our study. As controls, we selected only patients with CIP prophylaxis in order for the group to be as homogenous as possible for comparison. All patients underwent a standardized prostate biopsy procedure, which can be considered another strength of this study. Limitations of the study are the retrospective nature and the lack of a centralized microbiological laboratory. However, all laboratories involved in this study followed the same procedure of bacterial identification and the same EUCAST guidelines for antibiotic sensibility evaluation. We therefore consider the limitations of our study to be of minor importance for the outcome.

\section{Clinical implications}

Losing fluoroquinolones as first-choice antibiotics for prophylaxis in prostate biopsies may have significant implications, not only because diagnosis and treatment of prostate cancer depends on prostate biopsy as a safe procedure, but also because no other oral antibiotics can replace fluoroquinolones, except FT. Besides resistance to fluoroquinolones, Gram-negative uropathogens often show parallel resistance to trimethoprim/sulfamethoxazole and betalactams, including third generation cephalosporins and broadspectrum penicillins, even if combined with betalactamase inhibitors (BLI), such as amoxicillin/clavulanic acid [27]. Thus, alternatives to CIP easily involve intravenous broadspectrum antibiotics, which are not recommended for prophylaxis according to the EAU guidelines. Prolonged antibiotic prophylaxis extending beyond $24 \mathrm{~h}$ is also not in line with the EAU guidelines. The 5-day course of CIP as was given in our study might have been of benefit for some individual patients but might cause collateral damage to others and should be avoided according to the principles of antibiotic stewardship [17, 30]. Therefore, a prospective, randomized study with a peri-procedural one-day dosing of FT as used in the present retrospective study, as compared to a single dose of CIP would be highly recommended. The increasing number of men with prostate cancer on active surveillance is correlated with a higher rate of repeat biopsies that have a higher risk of infectious complications than primary biopsies [30]. Moreover, the finding that fluoroquinolone resistant and extended-spectrum betalactamases (ESBL)-producing isolates were the most commonly identified organisms in their cohort of patients highlights the need for appropriate information of surveillance patients about the increased risks associated with repeat prostate biopsies and the need for a thoughtful and tailored antibiotic prophylaxis [30].

\section{Conclusions}

In conclusion, FT appeared to be an attractive alternative antibiotic for peri-interventional prophylaxis in patients undergoing TR-PB due to a low rate of ADRs and a low 
rate of bacterial resistance against FT without cross- or parallel-resistance to other frequently used antibiotics.

Acknowledgments We are grateful to all members of the microbiological departments in all centers for their assistance and to Professor John Denton for manuscript language revision.

Authors' contribution Cai T. and Gallelli L. contributed to study conception and design; Cocci A., Tiscione D., Verze P., Lanciotti M., Vanacore D., Rizzo M., Gacci M., Saleh O., Damiano R., Palmieri A., Malossini G. and Liguori G helped in the acquisition of data; Cai T., Verze P. and Tiscione D. analyzed and interpreted the data; Cai T. and Gallelli L. drafted the manuscript; Wagenlehner F., Naber K., Mirone V. and Bjerklund Johansen T.E.is acknowledged for critical revision and supervisions; and Bjerklund Johansen T.E., Naber K., Bartoletti R., Mirone V., Carini M. and Trombetta C. supervised the intellectual concept.

\section{Compliance with ethical standards}

Conflict of interest Tommaso Cai has received grant from Zambon as speaker at scientific meeting; Kurt Naber and Florian Wagenlehner are consultant for and have received research support and grant as speakers at scientific meeting from Zambon.

\section{References}

1. Chun FK, Epstein JI, Ficarra V et al (2010) Optimizing performance and interpretation of prostate biopsy: a critical analysis of the literature. Eur Urol 58:851-864

2. Wagenlehner FM, van Oostrum E, Tenke P et al (2013) Infective complications after prostate biopsy: outcome of the Global Prevalence Study of Infections in Urology (GPIU) 2010 and 2011, a prospective multinational multicentre prostate biopsy study. Eur Urol 63(3):521-527

3. Cai T, Verze P, Bartoletti R et al (2015) Infectious complications after prostate biopsy: Time to rethink our clinical practice. World J Clin Urol 4(2):78-82

4. Zani EL, Clark OA, Rodrigues Netto N Jr (2011) Antibiotic prophylaxis for transrectal prostate biopsy. Cochrane Database Syst Rev 5:CD006576

5. Nam RK, Saskin R, Lee Y et al (2010) Increasing hospital admission rates for urological complications after transrectal ultrasound guided prostate biopsy. J Urol 183:963-968

6. Carignan A, Roussy JF, Lapointe V et al (2012) Increasing risk of infectious complications after transrectal ultrasound-guided prostate biopsies: time to reassess antimicrobial prophylaxis? Eur Urol 62(3):453-459

7. Bartoletti R, Cai T (2013) Prostate biopsies should be performed according to a standard of care. Eur Urol 63:528-529

8. Falagas ME, Kastoris AC, Kapaskelis AM, Karageorgopoulos DE (2010) Fosfomycin for the treatment of multidrug-resistant, including extended-spectrum beta-lactamase producing, Enterobacteriaceae infections: a systematic review. Lancet Infect Dis 10:43-50

9. Wagenlehner FM, Thomas PM, Naber KG (2014) Fosfomycin trometamol $(3000 \mathrm{mg})$ in perioperative antibiotic prophylaxis of healthcare-associated infections after endourological interventions: a narrative review. Urol Int 92(2):125-130

10. Rhodes NJ, Gardiner BJ, Neely MN et al (2015) Optimal timing of oral fosfomycin administration for pre-prostate biopsy prophylaxis. J Antimicrob Chemother 70(7):2068-2073
11. Clavien PA, Barkun J, de Oliveira ML et al (2009) The ClavienDindo classification of surgical complications: five-year experience. Ann Surg 250:187-196

12. Definition of historic cohort study-NCI Dictionary of Cancer Terms. http://www.cancer.gov/publications/dictionaries/ cancer-terms?CdrID $=286525$

13. European Association of Urology Guidelines on Prostate Cancer, update 2014. http://uroweb.org/wp-content/uploads/1607-Prostate-Cancer_LRV3.pdf

14. Testa G, Cacciatore F, Galizia G et al (2009) Charlson Comorbidity Index does not predict long-term mortality in elderly subjects with chronic heart failure. Age Ageing 38(6):734-740

15. Ongün S, Aslan G, Avkan-Oguz V (2012) The effectiveness of single-dose fosfomycin as antimicrobial prophylaxis for patients undergoing transrectal ultrasound-guided biopsy of the prostate. Urol Int 89(4):439-444

16. Horan TC, Gaynes RP (2004) Surveillance of nosocomial infections. In: Mayhall CG (ed) Hospital epidemiology and infection control, 3rd edn. Lippincott, Williams and Wilkins, pp 1659-1702

17. Zaytoun OM, Vargo EH, Rajan R et al (2011) Emergence of fluoroquinolone-resistant Escherichia coli as cause of postprostate biopsy infection: implications for prophylaxis and treatment. Urology 77:1035-1042

18. European Association of Urology Guidelines on Urological Infections. 2014. http://uroweb.org/wp-content/uploads/19-Urological-infections_LR2.pdf

19. Hooton TM, Scholes D, Gupta K et al (2005) Amoxicillin-clavulanate vs ciprofloxacin for the treatment of uncomplicated cystitis in women: a randomized trial. JAMA 293:949-955

20. http://www.eucast.org/clinical_breakpoints/

21. Badia X, Garcia-Losa M, Dal-Re R (1997) Ten-language translation and harmonization of the International Prostate Symptom Score: developing a methodology for multinational clinical trials. Eur Urol 31(2):129-140

22. Rosen RC, Riley A, Wagner G et al (1997) The international index of erectile function (IIEF): a multidimensional scale for assessment of erectile dysfunction. Urology 49(6):822-830

23. Gallelli L, Ferreri G, Colosimo M et al (2002) Adverse drug reactions to antibiotics observed in two pulmonology divisions of Catanzaro, Italy: a six-year retrospective study. Pharmacol Res 46(5):395-400

24. Naranjo CA, Busto U, Sellers EM et al (1981) A method for estimating the probability of adverse drug reactions. Clin Pharmacol Ther 30(2):239-245

25. Wolf JS Jr, Bennett CJ, Dmochowski RR et al (2008) Best practice policy statement on urologic surgery antimicrobial prophylaxis. J Urol 179:1379e90

26. Loeb S, Carter HB, Berndt SI, Ricker W, Schaeffer EM (2011) Complications after prostate biopsy: data from SEER-Medicare. J Urol 186:1830e4

27. Rhodes NJ, Gardiner BJ, Neely MN et al (2015) Optimal timing of oral fosfomycin administration for pre-prostate biopsy prophylaxis. J Antimicrob Chemother 70(7):2068-2073

28. Periti P, Novelli A, Reali EF, Lamanna S, Fontana P et al (1987) Prophylactic chemotherapy with fosfomycin trometamol salt during transurethral prostatic surgery: a controlled multicentre clinical trial. Eur Urol 13(suppl 1):122-131

29. Lista F, Redondo C, Meilán E et al (2014) Efficacy and safety of fosfomycin-trometamol in the prophylaxis for transrectal prostate biopsy. Prospective randomized comparison with ciprofloxacin. Actas Urol Esp 38(6):391-396

30. Tandogdu Z, Cek M, Wagenlehner F et al (2014) Resistance patterns of nosocomial urinary tract infections in urology departments: 8-year results of the global prevalence of infections in urology study. World J Urol 32(3):791-801 